\title{
Comparative evaluation of phenol and thimerosal as preservatives for a candidate vaccine against American cutaneous leishmaniasis
}

\author{
Wilson Mayrink¹, Carlos Alberto Pereira Tavares², Rosangela Barbosa de Deus ${ }^{3}$, \\ Melina Barros Pinheiro4, Tânia Mara Pinto Dabés Guimarães ${ }^{4}$, Hélida Monteiro de Andrade, \\ Carlos Alberto da Costa ${ }^{4}$, Vicente de Paulo Coelho Peixoto de Toledo ${ }^{4}+$
}

'Departamento de Parasitologia ${ }^{2}$ Departamento de Bioquímica e Imunologia, Instituto de Ciências Biológicas ${ }^{4}$ Departamento de Análises
Clinicas e Toxicológicas, Faculdade de Farmácia, Universidade Federal de Minas Gerais, Av. Antônio Carlos 6627, 31270-901 -
Belo Horizonte, MG, Brasil ${ }^{3}$ Departamento de Farmácia, Escola de Farmácia, Universidade Federal de Ouro Preto, Ouro Preto, MG, Brasil

For decades thimerosal has been used as a preservative in the candidate vaccine for cutaneous leishmaniasis, which was developed by Mayrink et al. The use of thimerosal in humans has been banned due to its mercury content. This study addresses the standardization of phenol as a new candidate vaccine preservative. We have found that the proteolytic activity was abolished when the test was conducted using the candidate vaccine added to merthiolate (MtVac) as well as to phenol (PhVac). The Montenegro's skin test conversion rates induced by MtVac and by PhVac was $68.06 \%$ and $85.9 \%$, respectively, and these values were statistically significant $(p<0.05)$. The proliferative response of peripheral mononuclear blood cells shows that the stimulation index of mice immunized with both candidate vaccines was higher than the one in control animals $(p<0.05)$. The ability of the candidate vaccines to induce protection in C57BL/10 mice against a challenge with infective Leishmania amazonensis promastigotes was tested and the mice immunized with PhVac developed smaller lesions than the mice immunized with MtVac. Electrophoresis of phenol-preserved antigen revealed a number of proteins, which were better preserved in PhVac. These results do in fact encourage the use of phenol for preserving the immunogenic and biochemical properties of the candidate vaccine for cutaneous leishmaniasis.

Key words: leishmaniasis - candidate vaccine - phenol - merthiolate

Leishmaniasis currently threatens more than 350 million men, women and children in 88 countries around the world. It is estimated that 2 million new cases occur each year, with at least 12 million people presently infected worldwide (WHO 2002). The disease is endemic in Africa, Southwest Asia, the Middle East, Southern Europe and Central and South America (Sinha et al. 2005).

Leishmaniasis in general, but particularly cutaneous leishmaniasis is one of a few parasitic diseases that will most likely become controllable through the use of vaccines. Given the relatively simple life cycle and the fact that recovery from a primary infection renders the host resistant to subsequent infections, indicates that a successful vaccination is feasible. During the past several decades, extensive efforts have been made to search for an effective Leishmania candidate vaccine (Khamesipour et al. 2006). Despite several efforts towards candidate vaccine development, no effective recombinant vaccine currently exists in humans. The induction of protective immunity using either inactivated or attenuated live vaccines (first generation vaccines) would be a significant step towards controlling the disease

\footnotetext{
Financial support: FAPEMIG

+ Corresponding author: vtoledo@ufmg.br

Received 3 August 2009

Accepted 1 December 2009
}

The Brazilian researcher, Mayrink and his coworkers conducted trials of different preparations of killed parasites in the 1970s. They developed a pentavalent candidate vaccine, which after two intramuscular injections was able to convert the Montenegro's leishmanin skin test [also known as Montenegro skin test (Mst)], resulting in the successful vaccination of $78.4 \%$ of volunteers within three months (Mayrink et al. 1979). However, the difficulties of standardization and establishing the safety of this candidate vaccine are potential obstacles to its registration.

In 2006, permission was granted (25000.045522/9910) by the Brazilian National Health Surveillance Agency (Brazil) to use the leishmaniasis candidate vaccine developed by Mayrink et al. (1979) as a therapeutic agent in the treatment of leishmaniasis. It is still used in Brazil and some other countries in Central America in its original formulation with thimerosal.

Recently, another point has been raised concerning the candidate vaccine stability. For decades, thimerosal - the most important preservative in vaccines and other injectable biological products - has been marketed mainly as a vaccine preservative. It remained in US pharmaceutical products until the 1980s when thimerosal began to be withdrawn because of its toxicity in topical pharmaceutical products. Ironically, while thimerosal was being eliminated from tropical countries, it was becoming more ubiquitous in the immunization schedule for infants and pregnant women. So far, there has not been a complete removal of thimerosal from several vaccines in US and today it also remains in numerous vaccines in Brazil (Geier et al. 2007). 
This study investigated the standardization of phenol as a new preservative for the anti-leishmaniasis candidate vaccine to replace thimerosal. It is critical to know the effect of proteolysis on immunogenicity and to determine the stability of different preparations of a singlestrain killed candidate vaccine conserved by thimerosal and phenol and whether phenol can stabilize candidate vaccine proteins more effectively than thimerosal. If so, it may be advisable to license the candidate vaccine as a therapeutic agent using the new proposed formulation with phenol as a preservative.

\section{SUBJECTS, MATERIALS AND METHODS}

Study subjects - A group of 247 Mst negative healthy subjects were immunized against American tegumentar leishmaniasis (ATL) using either merthiolated (mtVac) or phenolated (PhVac) candidate vaccine preparations. One hundred and nineteen individuals received the merthiolated preparation and 128 received the phenolated preparation. All of the study subjects resided in the endemic area of ATL in Caratinga, Minas Gerais, Brasil. This research was registered in the Conselho Nacional de Saúde, Comissão Nacional de Ética em Pesquisa, Ministério da Saúde, Brasil (13,085 - 02/09/2007).

Preparation of candidate vaccines - The candidate vaccine preparations used in this study were produced using a well defined WHO reference Leishmania amazonensis strain (IFLA/BR/67/PH8) in the Departamento de Parasitologia, Instituto de Ciências Biológicas (ICB), Universidade Federal de Minas Gerais (UFMG), Belo Horizonte, Minas Gerais, Brazil, according to a methodology described in detail elsewhere (Mayrink et al. 1979, Antunes et al. 1986, De Luca et al. 1999, Armijos et al. 2004). Briefly, promastigotes of L. amazonensis IFLA/BR/67/PH8 were grown in liver infusion medium and harvested at stationary phase (7 days of culture) using centrifugation. After washing with sterile saline, the pellet was divided in two: one part was disrupted by sonication and the other was then left particulated. The final concentration of total nitrogen was $240 \mu \mathrm{g} / \mathrm{mL}$. To preserve this candidate vaccine preparation, merthiolate was used in saline at $1: 10,000(\mathrm{v} / \mathrm{v})$ or a solution of phenol in saline at $0.35 \%(\mathrm{v} / \mathrm{v})$. After being produced, the candidate vaccines were stored at $4{ }^{\circ} \mathrm{C}$. All candidate vaccines used had been stored for four months.

Volunteer vaccination - Two different groups of volunteers were vaccinated with $\mathrm{mtVac}$ and PhVac in two $1.5-\mathrm{mL}$ doses injected intramuscularly in 21 day intervals. All the volunteers were Mst-negative and all the personnel involved in the trial were blinded with regard to which candidate vaccine preparation was used.

Mst - All vaccinated volunteers were skin-tested twice: two days before the administration of the first candidate vaccine dose (for screening of volunteers to be vaccinated) and 40 days after the administration of the second dose. The antigen was composed of the same strain used in the candidate vaccine preparation [L. amazonensis strain (IFLA/BR/67/PH8)] according to Costa et al. (1996). The test result was considered positive when induration with a diameter greater than or equal to $5 \mathrm{~mm}$ was detected $48 \mathrm{~h}$ after the injection.

Mice - Female C57BL/10 mice (8-12 week old) were obtained from the breeding facilities at the ICB-UFMG.

Immunization and challenge of mice - Forty isogenic C57BL/10 mice per group were vaccinated according to Costa et al. (1992). Twenty animals received two subcutaneous inoculations in seven-day intervals. Each dose contained $100 \mathrm{mg}$ of $\mathrm{mtVac}$ plus $250 \mathrm{mg}$ of Corynebacterium parvum as adjuvant. The other 20 animals received the phVac according to the same vaccination scheme. Twenty-eight days after the second dose, the animals received an additional $10 \mathrm{mg}$ of candidate vaccine without adjuvant. Seven days after the booster, 10 mice from both groups were challenged with $1 \times 10^{5} \mathrm{~L}$. amazonensis promastigotes inoculated subcutaneously into the base of the tails. These animals were examined at two-week intervals for a total of 180 days after the challenge to observe the appearance of lesions and their subsequent development and for measurement of lesion diameters. Animals were considered protected when no lesions were observed. The control group consisted of 10 other mice immunized with $C$. parvum alone. The 10 remaining vaccinated and unchallenged mice in each group were kept non-infected to be used in the evaluation of a lymphoproliferative response. All the animals reserved for this study were sacrificed and their spleens were removed aseptically to obtain the lymphocytes used in the lymphoproliferation assay.

Lymphocyte proliferation assay - The lymphocyte proliferation assay were performed as described by Costa et al. (1992). Briefly, spleen cells were harvested in a RPMI 1640 (SIGMA-USA) medium containing 10\% fetal calf serum (SIGMA, USA), 2 Mm L-Glutamine, 50 $\mathrm{mM}$ 2- $\beta$-Mercaptoethanol, 10,000 units of penicillin, 10 $\mathrm{mg}$ streptomycin and $250 \mathrm{mg}$ fungizone (SIGMA-USA) per $\mathrm{mL}$. Red blood cells were removed by lysis in 0.144 $\mathrm{mM} \mathrm{NH}_{4} \mathrm{Cl}$. The cells $\left(4 \times 10^{5} /\right.$ well $)$ were then incubated in triplicate in 96-well plates for five days either with $20 \mathrm{mg} / \mathrm{mL}$ of each L. amazonensis antigen or $0.5 \mu \mathrm{g} /$ $\mathrm{mL}$ of Concanavalin A (SIGMA, USA) in an atmosphere of $5 \% \mathrm{CO}_{2}$ at $37^{\circ} \mathrm{C}$. The antigens used in the proliferative assays were obtained by the same candidate vaccine preparation procedures (De Luca et al. 1999, Armijos et al. 2004). The antigen concentration was adjusted to 1 $\mathrm{mg} / \mathrm{mL}$. The proliferation was assayed by $[3 \mathrm{H}]$ thymidine incorporation $(0.2 \mathrm{mCi} /$ well, specific activity 5.0 $\mathrm{mCi} / \mathrm{mL}$ - Dupont, NEN Research Products, USA). The results were expressed as a stimulation index. A positive response showed a stimulation index equal to or higher than 2.5 (Mendonça et al. 1986).

Evaluation of proteolytic activity - Candidate vaccines produced with thimerosal or phenol were centrifuged at $100,000 \mathrm{~g}$ for $30 \mathrm{~min}$ at $4^{\circ} \mathrm{C}$. The supernatant was separated and the total nitrogen protein was estimated. The soluble fraction had its protease activity tested according to Erlanger et al. (1961). Briefly, $40 \mu \mathrm{g}$ of protein of each candidate vaccine and $100 \mu \mathrm{g}$ of trypsin were mixed and 
the volume was brought to $1 \mathrm{~mL}$ with $0.075 \mathrm{M}$ Tris-HCL, $\mathrm{pH}$ 7.6, containing $25 \mathrm{mM} \mathrm{CaCl}$. After incubation at $30^{\circ} \mathrm{C}$ for $15-120 \mathrm{~min}$ with the synthetic substrate, Bapna (N-benzoyl-L-arginine-p-nitroanilide), at a final concentration of $1 \times 10^{-5} \mathrm{M}$, the reaction was stopped with the addition of $60 \%$ acetic acid. The extent of hydrolysis was measured by a spectrophotometer (Shimadzu) at $405 \mathrm{~nm}$; each sample was assayed in triplicate.

Electrophoresis of candidate vaccine antigens Twenty micrograms of antigen were submitted to electrophoresis in a $10 \%$ polyacrilamide gel (SDS-PAGE) (Laemmli 1970). Then the gel was fixed overnight in $50 \%$ methanol and stained using the silver nitrate method (Merril et al. 1981).

Statistical analysis - One way ANOVA was used for the evaluation of proteolytic activity in mtVac and phVac experiments and for the lymphocyte proliferation assay. To analyze responses to Mst, the Mann-Whitney test was employed. For the immunization and challenge of mice, we used bootstrapping with a probability function, according to the central limit theorem to assume normality followed by means comparison by one way ANOVA.

\section{RESULTS}

Mst - The volunteers who received the mtVac had a mean age of $28.89 \pm 20.45$, while the phenol vaccinated volunteers had a mean age of $30.32 \pm 21.41$. All study subjects had negative results for the first skin test. The induration area of positivity for the second skin test in the groups who had received either mtVac and phVac is shown in $\mathrm{mm}^{2}$ in the Table. The Mst rates induced by $\mathrm{mtVac}$ and by phVac were $68.06 \%$ and $85.9 \%$, respectively, and these values were statistically significant $(\mathrm{p}<0.05)$. No difference was found between the average vertical and transversal diameters of the induration area $(\mathrm{p}>0.05)$.

Evaluation of immunoprotection in mice - The ability of the candidate vaccines to induce persistent protection in C57BL/10 mice against a challenge with infective L. amazonensis promastigotes was tested (Fig. 1). Mice were immunized with each candidate vaccine according to the schedule described in the materials and methods. The animals were examined for the appearance of lesions at the site of inoculation and/or other manifestations along the course of 180 days. Animals were considered protected when no lesions were observed. As shown in Fig. 1, all the control mice developed a progressive infection at the base of the tail consisting of ulceration and necrosis between the 7th-17th weeks after the challenge. Along the same time range, the immunized mice with phenolated vaccine developed smaller lesions than the mice immunized with mtVac. Around week 17 after the challenge, about 50\% of the immunized mice from both groups developed lesions, which were about 10 times smaller than those in the control group. No visceralization was observed in either the control or vaccinated animals.

Lymphocyte proliferation assay - Spleen cells from each immunized and control group were incubated with the homologous candidate vaccine antigens. The pro-
TABLE

Montenegro's skin test (Mst) conversion rates after vaccination

\begin{tabular}{lcc}
\hline Candidate vaccines & $\begin{array}{c}\text { Mst conversion rate } \\
(\text { average })^{a}(\%)\end{array}$ & $\begin{array}{c}\text { Average induration area } \\
\left(\mathrm{mm}^{2}\right)\end{array}$ \\
\hline $\mathrm{mtVac}$ & $68.06(81 / 119)$ & 50.23 \\
phVac & $85.9(110 / 128)$ & 62.84 \\
\hline
\end{tabular}

$a$ : significant difference between the merthiolated $(\mathrm{mtVac})$ and phenolated (phVac) candidate vaccines $(\mathrm{p}<0.05)$.

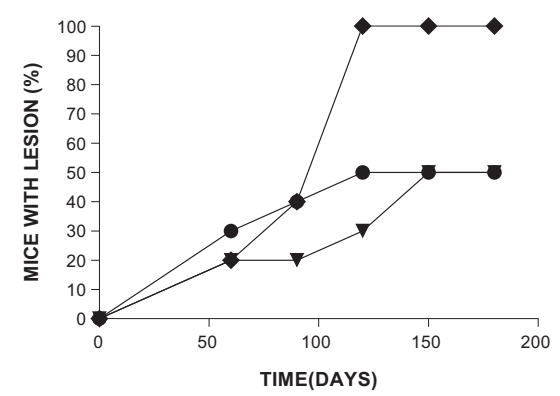

Fig. 1: the course of Leishmania amazonensis infection in control C57BL/10 mice ( $\downarrow$ ) and mice immunized with phenolated candidate vaccine plus Corynebacterium parvum $(\boldsymbol{\nabla})$ or with merthiolated candidate vaccine plus $C$. parvum $(\bullet)$. The adjuvant $C$. parvum was included in all immunizations. The control group consisted of mice immunized with C. parvum alone (all the control animals developed lesions). The data are the mean of 10 vaccinated mice per group.

liferative response was measured by $[3 \mathrm{H}]$ - thymidine incorporation. Fig. 2 shows that the stimulation index of mice immunized with candidate vaccine antigens was at least 4.9 times higher than the one in control animals (non-vaccinated) and this proliferative response was significantly higher $(p<0.05)$. The control consisted of nonvaccinated mouse spleen cells stimulated with candidate vaccine antigens. No difference was observed between the groups ( $\mathrm{mtVac}$ and phVac) in this regard. All the ConA-stimulated spleen cells from the non-vaccinated mice showed a stimulation index above 7.0, suggesting that the culture cell system was sufficiently viable.

Evaluation of proteolytic activity - Fig. 3 shows that the test for determining the enzymatic activity was efficient because both the controls of substrate and the enzyme trypsin (TryC) had the expected results. The protease activity in the Leishmania extract (strain PH8) was similar to that seen in the control enzyme (TryC), which was expected because the preparation, in addition to being recently prepared, had no protease inhibitor added. We also found that the proteolytic activity was abolished when the test was conducted using MtVac or PhVac.

Electrophoresis of candidate vaccine antigens - A reducing SDS-PAGE was performed on a slab gel apparatus with the discontinuous system. The separated protein bands were stained using silver stain. In Fig. 4, 


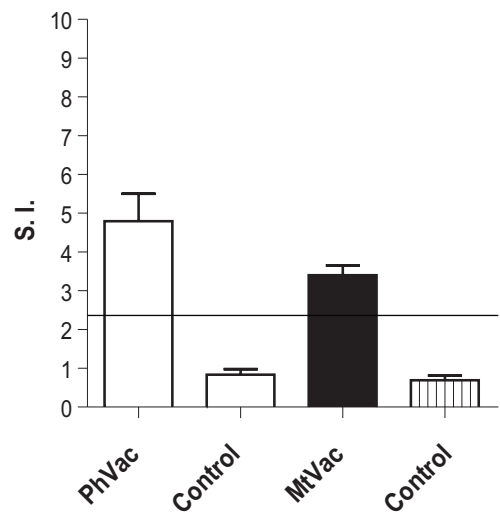

Fig. 2: the lymphoproliferative response (shown as a stimulation index) of spleen cells from mice immunized with the phenolated (PhVac) and merthiolated $(\mathrm{MtVac})$ candidate vaccines after in vitro stimulation with homologous antigen. Spleen cells from non-vaccinated mice were used as the control and were stimulated with phVac antigens or $\mathrm{mtVac}$ antigens. The stimulation indexes greater than or equal to 2.5 were considered positive (horizontal line).

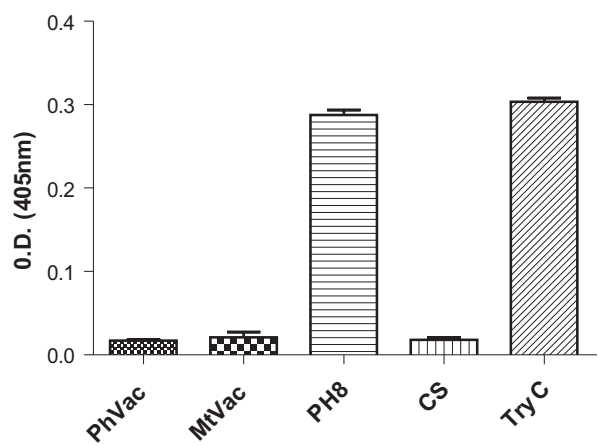

Fig. 3: evaluation of proteolytic activity using BApNA as substrate. One hundred micrograms of protein was used for the control for trypsin activity (TryC). The vertical lines represent the standard deviation for each analysis. The data are the mean of three experiments. The top bar refers to SD. CS: control for substrate activity; MtVac: merthiolated candidate vaccine; PhVac: phenolated candidate vaccine; PH8: fresh extract from Leishmania.

qualitative and quantitative differences were observed in the total protein profiles of the two samples. The electrophoresis of phenol-preserved antigen revealed a number of proteins, including those already described in the literature as immunogenically more immunogenic: gp63, gp46 and a 30-kDa protein, among others (Nascimento et al. 1990). Protein degradation was more evident in the merthiolated antigen since all of the above-mentioned antigens were not as evident as in the phVac. Moreover, the merthiolated antigen had fewer bands and they were more diffuse.

\section{DISCUSSION}

Therapeutic trials of first generation leishmaniasis candidate vaccines have shown very encouraging results. From Convit's trials for the treatment of patients in Vene- zuela and Machado Pinto's results in Brazil, to the results of Musa et al. (2008) on therapy of post Kalazar dermal leishmaniasis in Sudan, immunochemotherapy seems a promising mode of treatment (Convit et al. 1987, 2003, Machado Pinto et al. 2002, Musa et al. 2008). This justifies further investigation of first generation leishmaniasis candidate vaccines for therapeutic purposes. The accumulated evidence suggests that protective immunity followed by healing is attributed to the development of a strong Leishmania-specific $\mathrm{CD}^{+}$Th1 cell response, which produces and releases Th1 cytokines, mainly Interferon- $\gamma$, which has the ability of activating macrophages to inhibit and/or kill parasites (Amaral et al. 2002).

In our study, we assessed the ability of a candidate vaccine, preserved by phenol, to induce immunity against Leishmania. Thus, we measured delayed hypersensitivity to the Mst antigen and evaluated the spleen cell proliferative response. In trials where post vaccination Mst was measured, responses were notably larger in the candidate vaccine group, but this evidence of immunogenicity induced by the candidate vaccine was not carried over to having a protective effect. Nevertheless, conversion from a negative Mst reaction to Mst > $5 \mathrm{~mm}$ after vaccination has been observed to be associated with a significantly lower incidence of infection in Brazil, Iran and Sudan, suggesting that Mst conversion may be associated with an immune response that provides some level of protection (Noazin et al. 2008). The percentage of Mst conversion is essentially the average described by Mayrink et al. (1978), which was $78.4 \%$. In our study, the subjects were examined by the study clinicians on the day of vaccination and on post-vaccination days for the presence of lymphadenopathy, fever, ulcerations, redness, bleeding and swelling at the vaccination site. No major side effects were registered in the volunteers who received phVac or $\mathrm{mtVac}$. No individual aspects of the subjects or possible correlations with age, gender or nutritional status were significantly different based on variance analysis.

A skin test conversion was observed in the majority of vaccinated volunteers. The conversion rates induced by phVac and $\mathrm{mtVac}$ were $85.9 \%$ and $69 \%$, respectively, and this difference was statistically significant $(\mathrm{p}<0.03)$. The Mst demonstrates the immunogenicity of the monostrain PH8 candidate vaccine, independent of the type of preservative used. We can infer that the higher Mst conversion rate in the group that received phVac (85.9\%) could be explained by the improved preservation of antigens, as observed from the vaccines protein silver stain gel (Fig. 4). Therefore, we can rule out the possibility of hypersensitivity to phenol reactogenicity, since Mayrink et al. (2006) demonstrated its absence in Montenegro's antigens preserved by phenol as well as in merthiolate. Furthermore, in both groups the averages of the Mst induration diameters were not statistically significant.

In regard to the spleen cell proliferative response, our data show that both candidate vaccines elicit an antigenspecific recall response in spleen cells from mice immunized with these candidate vaccines. Compared to 


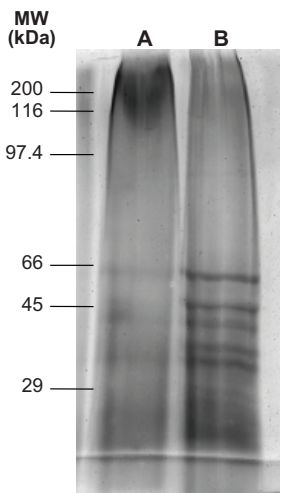

Fig. 4: electrophoresis in $10 \%$ polyacrilamide gel silver stained using merthiolated and phenolated candidate vaccines. Lane A: protein profile for merthiolated-preserved antigens: B: protein profile for phenolpreserved antigens. MW molecular weight markers $(\mathrm{kDa})$.

the control groups, there was a significant increase in the antigen-stimulated spleen cell proliferative response in vaccinated mice, demonstrating the immunogenicity of both candidate vaccines. Thus, merthiolate and phenol preserve the immunogenicity of the Leishmania antigens in this single strain candidate vaccine. To supplement this investigation, we also investigated the immunogenicity and efficacy of an L. amazonensis candidate vaccine. Groups of C57BL/10 mice were immunized with both candidate vaccines plus $C$. parvum as an adjuvant. Both $\mathrm{mtVac}$ and phVac induced protective immunity against infective promastigotes. The animals presenting lesions of any size were considered non-protected, but the lesions observed in the vaccinated, nonprotected mice were always much smaller than those in the unvaccinated animals. No self healing was observed in the control or non-protected mice. Interestingly, near week 17 the lesions in the group that received the phVac had a delayed onset and a smaller size than those in the group that received $\mathrm{mtVac}$. The data obtained from this experiment, combined with the significant increase in the antigen-stimulated spleen cell proliferative response after vaccination demonstrate the immunogenic efficacy of the single strain candidate vaccine.

The current results confirm those conducted by other groups working with candidate vaccines formulated with different Leishmania strains that showing the immunogenicity of the candidate vaccine is maintained with both merthiolate and phenolate preservatives (Mayrink et al. 1979, Mendonça et al. 1995, Armijos et al. 1998, 2004).

The antigen stability of these candidate vaccines was analyzed by polyacrilamide gel electrophoresis. We observed the same number of bands in both the mtVac and phVac, but the latter were more intense. This phenomenon was also observed in Mst when it was conserved by phenol (Mayrink et al. 2006).

Thimerosal not only helps to prevent the growth of bacteria and fungi in vaccines but can also prevent proteolysis. According to the protease activity test (Fig. 3), both MtVac and PhVac present low protease activity when compared to TryC and to a Leishmania extract without protease inhibitor. These data demonstrate that candidate vaccines prepared with merthiolate or phenol do not suffer from degradation by proteolytic enzymes, indicating these substances are efficient for preserving vaccines.

Tests conducted with the soluble fraction of candidate vaccines showed that the phVac had its proteolytic activity reduced by $90 \%$ and the $\mathrm{mtVac}$ by $34 \%$. This result indicates that phenol is a more efficient preservative agent than merthiolate (data not shown).

A meningococcal vaccine stabilized with $0.25 \%$ of phenol was reported to be stable for two years (WHO 1981). Considering the diminished protease activity through the use of phenol in leishmaniasis candidate vaccine, we expect a similar storage time for a phenolated leishmaniasis candidate vaccine.

Taken together, this study encourages the use of phVac as a means for preserving the immunogenic and biochemical properties of leishmaniasis candidate vaccine.

\section{ACKNOWLEDGEMENTS}

To the Secretaria Municipal de Saúde of Caratinga, state of Minas Gerais, Brazil, and Mr. Jair Cecílio de Paula, for his invaluable support during the field work.

\section{REFERENCES}

Amaral VF, Teva A, Oliveira-Neto MP, Silva AJ, Pereira MS, Cupolillo E, Porrozzi R, Coutinho SG, Pirmez C, Beverly SM, Grimaldi GJr 2002. Study of the safety, immunogenicity and efficacy of attenuated and killed Leishmania (Leishmania) major vaccines in a rhesus monkey (Macaca mulatta) model of the human disease. Mem Inst Oswaldo Cruz 97: 1041-1048.

Antunes CMF, Mayrink W, Magalhães PA, Costa CA da, Melo MN, Dias M, Michalick MSM, Williams P, Oliveira-Lima A, Vieira JBF, Schettini APM 1986. Controlled field trials of a vaccine against new cutaneous leishmaniasis. Int J Epidemiol 15: $147-154$.

Armijos RX, Weigel MM, Aviles H, Maldonado R, Racines J 1998. Field trial of a vaccine against New World cutaneous leishmaniasis in an at-risk child population: safety, immunogenicity and efficacy during the first 12 months of follow-up. J Infect Dis 177: 1352-1357.

Armijos RX, Weigel MM, Calvopina M, Hidalgo A, Cevallos W, Correa J 2004. Safety, immunogenicity and efficacy of an autoclaved Leishmania amazonensis vaccine plus BCG adjuvant against New World cutaneous leishmaniasis. Vaccine 22: 1320-1326.

Convit JO, Castellanos PIO, Rondon AO, Pinardi MEO, Ulrich MO, Castes M 1987. Immunotherapy versus chemotherapy in localised cutaneous leishmaniasis. Lancet 1: 401-405.

Convit JO, Ulrich MO, Zerpa OO, Borges RO, Aranzazu NO, Valera MO, Villarroel HO, Zapata Z, Tomedes I 2003. Immunotherapy of American cutaneous leishmaniasis in Venezuela during the period 1990-1999. Trans R Soc Trop Med Hyg 97: 469-472.

Costa CA da, Afonso LCC, Toledo VPCP, Guimarães TMPD, Nascimento E, Tavares CAP, Mayrink W 1992. Immune responses and protection induced in mice by an industrialized vaccine against American cutaneous leishmaniasis. Parassitologia 34: 45-51.

Costa CA da, Toledo VPCP, Genaro O, Williams P, Mayrink W 1996. Montenegro skin test evaluation of the composition and stability of the antigen preparation. Mem Inst Oswaldo Cruz 91: 193-194. 
De Luca PM, Mayrink W, Alves CR, Coutinho SG, Oliveira MP, Bertho AL, Toledo VPCP, Costa CA, Genaro O, Mendonça SC 1999. Evaluation of the stability and immunogenicity of autoclaved and non autoclaved preparations of a vaccine against American tegumentary leishmaniasis. Vaccine 17: 1179-1185.

Erlanger BF, Kokowsky N, Cohen W 1961. The preparation and properties of two new chromogenic substrates of trypsin. Arch Biochem Biophys 95: 271-278.

Geier DA, Sykes LK, Geier MR 2007. A review of thimerosal (merthiolate) and its ethylmercury breakdown product: specific historical considerations regarding safety and effectiveness. $J$ Toxicol Environ Health B Crit Rev 10: 575-596.

Khamesipour A, Rafati S, Davoudi N, Maboudi F, Modabber F 2006. Leishmaniasis vaccine for development: a global overview. Indian J Med Res 123: 423-438.

Laemmli UK 1970. Cleavage of structural protein. The assembly of the head of bacteriophage T4. Nature 27: 680-685.

Machado-Pinto J, Pinto J, da Costa CA, Genaro O, Marques MJ, Modabber F, Mayrink W 2002. Immunochemotherapy for cutaneous Leishmaniasis: a controlled trial using killed Leishmania (Leishmania) amazonensis vaccine plus antimonial. Int $J$ Dermatol 41: 73-78.

Mayrink W, Costa CA da, Magalhães PA 1979. A field trial of a vaccine against American dermal leishmaniasis. Trans $R$ Soc Trop Med Hyg 73: 385-387.

Mayrink W, Machado-Coelho GLL, Guimarães TMPD, Andrade HM, Perez EC, Costa CA da, Toledo VPCP 2006. Immuno-biochemical evaluations of phenol and merthiolate as antigens preservatives in Montenegro skin test. Acta Trop 98: 87-93.

Mayrink W, Magalhães PA, Dias M, Costa CA da, Melo MN, Oliveira-Lima A 1978. Responses to Montenegro antigen after immunization with killed Leishmania promastigotes. Trans $R$ Soc Trop Med Hyg 72: 676.
Mendonça SC, Coutinho SG, Amendoeira RR, Marzochi MC, Pirmez C 1986. Human American cutaneous leishmaniasis (Leishmania b. braziliensis) in Brazil: lymphoproliferative responses and influence of therapy. Clin Exp Immunol 64: 269-276.

Mendonça SCF, De Luca PM, Mayrink W, Restom TG, ConceiçãoSilva F, da-Cruz AM, Bertho AL, Costa CA da, Genaro O, Toledo VPCP, Coutinho SG 1995. Characterization of the human $\mathrm{T}$ lymphocyte-mediate immune response induced by a vaccine against American tegumentary leishmaniasis. Am J Trop Med Hyg 53: 195-201.

Merril CR, Goldman D, Sedman SA, Ebert MH 1981. Ultrasensitive strain for proteins in polyacrilamide gels shows regional variation in cerebrospinal fluid proteins. Science 211: 1437-1438.

Musa AM, Khalil EA, Mahgoub FA, Elgawi SH, Modabber FO, Elkadaru AE 2008. Immunochemotherapy of persistent post-kalaazar dermal leishmaniasis: a novel approach to treatment. Trans R Soc Trop Med Hyg 102: 58-63.

Nascimento E, Mayrink W, Costa CA da, Michalick MSM, Melo MN, Barros GC, Dias M, Antunes CMF, Lima MS, Taboada DC, Liu TY 1990. Vaccination of humans against cutaneous leishmaniasis: cellular and humoral immune responses. Infect Immun 58: 2198-2203.

Noazin S, Moddaber F, Khamesipour A, Smith PS, Moulton LH, Nasseri K, Sharifi I, Khalil EAG, Bernal IDV, Antunes CMF, Kieny MP, Tanner M 2008. First generation leishmaniasis vaccines: a review of field efficacy trials. Vaccine 26: 6759-6767.

Sinha PK, Pandey K, Bhattacharya SK 2005. Diagnosis \& management of Leishmania/HIV co-infection. Indian J Med Res 121: 407-414.

WHO 1981. Report of Expert Committee on Biological Standartization. Requirements for meningococcal vaccine. Technical Report Series 658. Available from: www.who.int/biologicals/publications/trs/areas/en/.

WHO 2002. Control of the leishmaniasis: report of a WHO Expert Committee. Report Series 793. Available from: www.who.int/ biologicals/publications/trs/areas/en/. 